$617-79$

\title{
equivalencia aproximada entre las resistencias a la compresión de los morteros de cemento Portland según los Pliegos españoles de 1930, 1960 y algunas Normas extranjeras
}

J. CALLEJA

\section{O. Introducción}

Cuando por razones de modernización y puesta al día de las prescripciones y normas de ensayo de un determinado material, por ejemplo el cemento, se cambian las especificaciones relativas al mismo, es de todo punto interesante conocer la equivalencia entre las resistencias mecánicas de un mismo cemento ensayado según los métodos antiguos que se abandonan y según los modernos que se adoptan.

Generalmente, la correlación buscada se tiene de antemano por vía experimental y estadística, pues la comparación en todos los aspectos entre los métodos viejos y los nuevos forma parte indispensable del estudio encaminado a justificar la implantación de estos últimos.

Así ha sucedido recientemente al sustituir el Pliego español de 1930 por el de 1960 , y con anterioridad, en 1939, al reemplazar la antigua Norma alemana por la DIN 1164.

Para establecer en cada caso la equivalencia por vía experimental, sería preeiso que los laboratorios de cada país estuviesen dotados de todos los medios precisos para realizar los ensayos según cualquier Norma $o$, cuando menos, según algunas de ellas.

Esto, que no es práctico (lo práctico sería que existiese una única Norma mundial), tampoco es posible en muchos casos, pues, si bien es fácil hasta cierto punto disponer de los equipos mecánicos necesarios, no lo es tanto disponer de las arenas normales nacionales prescritas por cada Norma.

$\mathrm{Al}$ establecimiento de una equivalencia aceptable se puede llegar también por vía teórica, aunque empírica, estableciendo una serie de hipótesis que de hecho no se cumplen sino de una manera aproximada. Sin embargo, la aproximación es suficiente, en general, dada la finalidad que se persigue en la mayor parte de los casos con dicha equivalencia.

* Para información detallada sobre el tema véase el trabajo completo del Dr. J. CaLlesa, titulado Equivalencia aproximada entre las resistencias a la compresión de los morteros de cemento Portland según las distintas Normas. Monografía núm. 211, I. T. C. C., Madrid, 1961, del cual lo que sigue es un breve resumen. 
Esto tiene un interés particular, tanto para el fabricante como para el usuario del cemento, cuando se pretende deducir los valores de la resistencia del mismo según los ensayos de una cierta Norma, conocidos los que da de acuerdo con los de otra cualquiera. Esto se precisa, en general, cuando por razones de exportación, o de la contrata de obras de envergadura (tanto de carácter militar como civil) por parte de empresas extranjeras, se exige al cemento de una determinada nacionalidad que cumpla los requisitos exigidos por una Norma de otra nacionalidad diferente.

Por ello, se suele recurrir al establecimiento de fórmulas empíricas apoyadas en hipótesis sencillas, que permiten calcular las equivalencias salvando el inconveniente que tiene la deducción de correlaciones basadas en estadísticas sobre datos experimentales, sujetas a revisión constante, y de poca consistencia, dado que, en rigor, sólo permiten comparar resistencias obtenidas por métodos muy análogos.

Estas fórmulas no van igualmente bien en todos los casos, pero cumplen con sui misión, ya que, en general, las tolerancias admisibles son amplias. En lo que sigue se trata de establecer una fórmula. deducida de la de Abrams, previa la introducción de ciertas modificaciones en ella.

\section{Fórmula de Abrams modificada}

De las muchas variables que influyen en la resistencia mecánica a la compresión de los morteros y hornigones: composición (relaciones árido/cemento y agua/cemento); sistemas de compactación y curado; tipo y tamaño de las probetas; naturaleza y gramulometría del árido, etc., hay una, la relación agua/cemento, que cuando las otras oscilan entre límites estrechos, puede considerarse como variable principal y casi única.

Esto es lo que sucede en los morteros de ensayo de las distintas Normas para cemento, pues ni la compactación y el curado, ni la forma y tamaño de las probetas, ni la naturaleza y granulometría de las arenas tipo, ni la dosificación, es decir, la relación cemento/arena, son tan distintas de unas Normas a otras como puede serlo la relación agua/cemento (morteros secos, plásticos o flúidos).

Admitida la relación agua/cemento como variable principal de la resistencia a la compresión de los hormigones, y por extensión de los morteros de ensayo, Abrams estableció una fórmula empírica para expresar la segunda en función de la primera:

$$
\mathrm{R}=\frac{\mathrm{A}}{\mathrm{B}^{\left(\mathrm{in} / \mathrm{v}_{\nu}\right.}}
$$

En esta fómula la relación agua/cemento, $(\mathrm{a} / \mathrm{c})_{v}$ está expresada en volumen (real o aparente de agua y aparente de cemento), y A y B son constantes empíricas que se obtienen experimentalmente; A engloba la influencia de las restantes variables señaladas, poco operantes, y B depende del cemento y representa un factor de calidad del mismo.

Para probetas cilíndricas de hormigón de $15 \mathrm{~cm}$ de diámetro y $30 \mathrm{~cm}$ de altura, hechas con cementos de calidad intermedia, siendo $\mathrm{R}$ la resistencia a la compresión a 28 días, los valores de A y $\mathrm{B}$ deducidos por Abrams son los siguientes:

$$
\mathrm{A}=980 \quad ; \quad \mathrm{B}=\operatorname{de} 7 \text { a } 9
$$

Si se expresa la relación agua/cemento en peso $(\mathrm{a} / \mathrm{c})_{\infty}$, teniendo en cuenta que la densidad aparente del cemento con un asentamiento normal es de 1,25 , y se considera que la resistencia de una probeta cúbica de $7 \mathrm{~cm}$ de arista es de 1,3 veces la de una cilíndrica de las dimensiones anteriores, la 
resistencia a la compresión a los 28 días de una probeta cúbica de $7 \mathrm{~cm}$, en función de la relación agua/cemento viene dada por:

$$
\mathrm{R}_{28}=1.274 / \mathrm{B}^{1,26}(\mathrm{a} \mid \mathrm{c})_{p}
$$

es decir:

$$
\log R_{28}=3,10517-1,25(a / c)_{p} \cdot \log B
$$

\section{Valores de B para los cementos españoles}

La relación agua/cemento del mortero de ensayo según el Pliego español de 1930 es variable, según el tanto por ciento de agua en la pasta de consistencia normal definida en el mismo. Dicha relación suele oscilar entre 0,32 y 0,36 según los cementos, pudiéndose considerar como valor medio 0,34 .

La relación agua/cemento del mortero de ensayo según el Pliego español de 1960 es fija e igual a 0,5 .

Con los valores de las resistencias a compresión a 28 dias y los de las respectivas relaciones agua/ cemento de numerosos morteros hechos con distintos cementos Portland y ensayados tanto por uno como por otro Pliego, se ha podido apreciar que el valor medio de $B$ oscila entre 8 y 8,4 , de acuerdo con el valor medio resultante de la fórmula de Abrams. Las distribuciones estadísticas de valores de $B$ fueron normales en todos los casos.

\section{Cálculo de la resistencia del cemento según una norma, conocida la que tiene según otra}

Dada la coincidencia entre el valor medio de $\mathbf{B}$ para los cementos Portiand españoles y el señalado por Abrams para los de tipo intermedio, en general, tal como queda expuesto en 2 , si son $R_{\mathrm{N}}$ y $r_{\mathrm{N}}$, y $\mathrm{R}_{\mathrm{N}}{ }^{\prime}$ y $r_{\mathrm{N}}{ }^{\prime}$ las resistencias a compresión a 28 días y las correspondientes relaciones agua/cemento de los morteros de ensayo según dos Normas distintas $\mathrm{N}$ y $\mathrm{N}^{\prime}$, se tiene, según [II] :

$$
\begin{aligned}
\log \mathrm{R}_{\mathrm{N}} & =3,10517-1,25 r_{\mathrm{N}} \cdot \log \mathrm{B} \\
\log \mathrm{R}_{\mathrm{N}}^{\prime} & =3,10517-1,25 r_{\mathrm{N}^{\prime}} \cdot \log \mathrm{B}
\end{aligned}
$$

es decir:

$$
\log \mathrm{B}=\frac{3,10517-\log \mathbf{R}_{\mathrm{N}}}{1,25 r_{\mathrm{N}}}=\frac{3,10517-\log \mathrm{R}_{\mathrm{N}}^{\prime}}{1,25 r_{\mathrm{N}}^{\prime}}
$$

de donde:

$$
\log \mathrm{R}_{\mathrm{N}}=3,10517-\frac{r_{\mathrm{N}}^{\prime}}{r_{\mathrm{N}}}\left(3,10517-\log \mathrm{R}_{\mathrm{N}}\right)
$$

Esta ecuación permite calcular la resistencia de un cemento $\mathrm{R}_{\mathrm{N}}{ }^{\prime}$, según la Norma $\mathrm{N}^{\prime}$ en función de su resistencia $R_{N}$ según la Norma $\mathrm{N}$, cuando se conocen las relaciones agua/cemento $r_{\mathrm{N}}, y r_{\mathrm{N}}$ del mismo en ambas Normas.

3.1. Relación entre las resistencias según los Pliegos españoles de 1930 y 1960.

En particular, de la relación [III], si N representa el Pliego español de 1930 (antiguo A) y N' el 
Pliego español de 1960 (moderno M), se tiene:

$$
\begin{aligned}
& \log \mathrm{R}_{3,}=3,10517 \cdots \frac{0,50}{r_{A}\left(3,10517-\log \mathrm{R}_{A}\right)} \\
& \log \mathrm{R}_{A}=3,10517 \cdots \frac{r_{A}}{0,50}\left(3,10517 \ldots \log \mathrm{R}_{M}\right)
\end{aligned}
$$

y si, según lo indicado en 2 , se considera para $r_{\mathrm{A}}$ el valor medio de 0,34 :

$$
\begin{aligned}
& \log \mathbf{R}_{31}=1,47 \log R_{A}-1,45943 \\
& \log R_{A}=0,68 \log R_{31}-0,99365
\end{aligned}
$$

Aplicadas estas ecuaciones a casos concretos, resultan resistencias calculadas que, por término medio, difieren de las experimentales en $\pm 10 \%$, tolerancia perfectamente admisible con fines prácticos.

Si se consideran los valores mínimos prescritos para las resistencias a compresión a 28 días por los Pliegos de 1930 y 1960, la relación entre éstos es la expuesta en los cuadros 1 y 2 , en los que se indican dichos valores redondeados a 0 ó 5 .

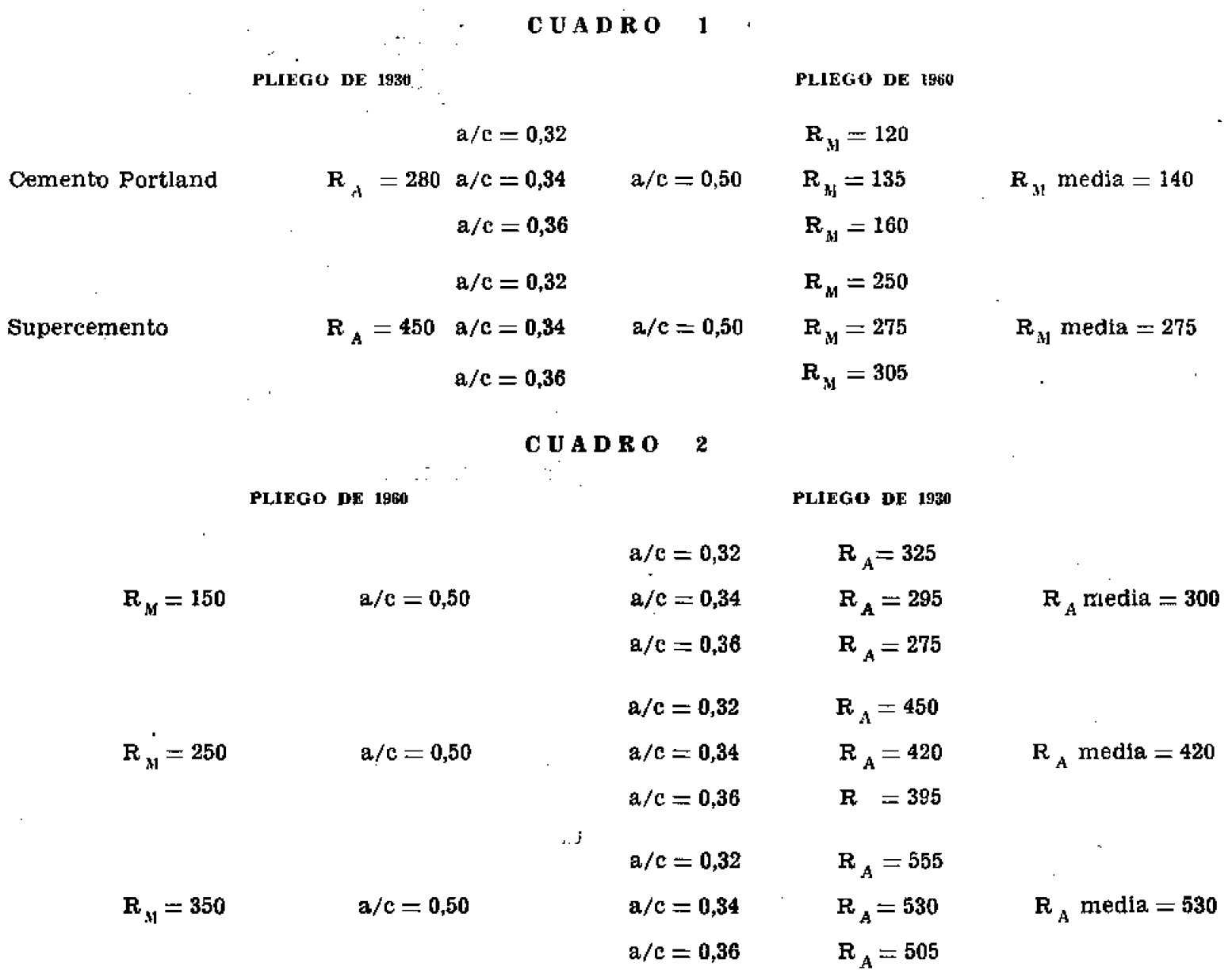

Con los valores de los cuadros 1 y 2 se ha construído el gráfico de la figura 1 , que expresa la equivalencia aproximada entre las resistencias a compresión a 28 días según los Pliegos españoles antiguo de 1930 y moderno de 1960. 


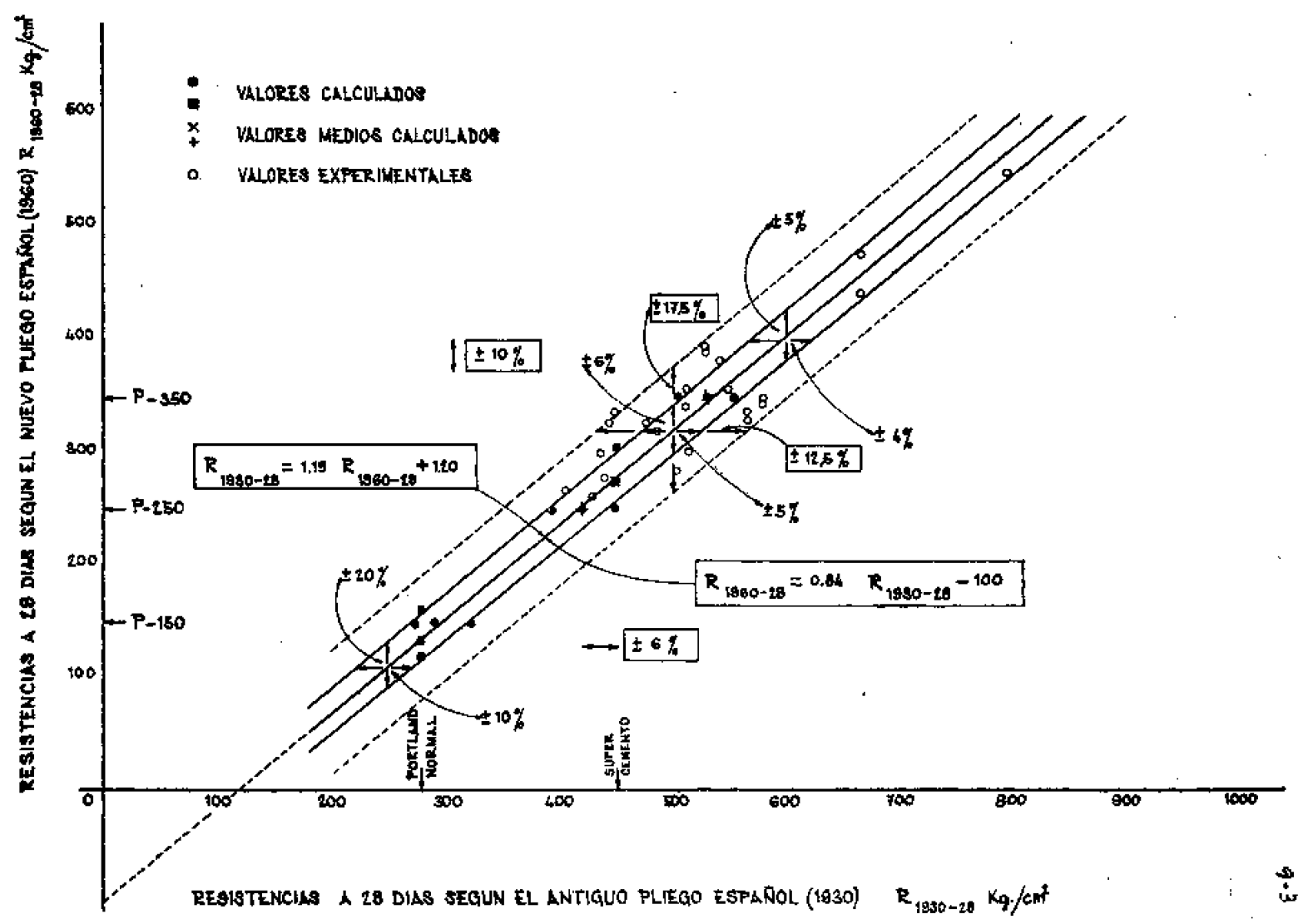

Fig. 1

Puede apreciarse en ella que las desviaciones entre los valores calculados a partir de la recta media $(a / c=0,34)$ y los valores experimentales (puntos sin rellenar) puede llegar a ser del orden de $\pm 15 \%$.

3.2. Relación entre las resistencias según los Pliegos españoles y según algunas Normas extranjeras.

La ecuación [III], general, es de aplicación en estos casos. Si la Norma $\mathrm{N}$, de cuyos datos se supone que se parte es el Pliego español antiguo o el moderno, dicha ecuación da lugar a otras dos:

$$
\begin{aligned}
& \log \mathbf{R}_{N^{\prime}}=3,10517-\frac{r_{N^{\prime}}}{0,34}\left(3,10517-\log \mathbf{R}_{A}\right) \\
& \log \mathbf{R}_{N^{\prime}}=3,10517-\frac{r_{N}^{\prime}}{0,50}\left(3,10517-\log \mathbf{R}_{M}\right)
\end{aligned}
$$

que permiten calcular en cada caso la resistencia de un cemento $\mathbf{R}_{\mathrm{N}}$, según una Norma $\mathbf{N}^{\prime}$, cuando se conoce su resistencia en uno de los Pliegos españoles, $1_{A} \circ R_{M}$, y la relación agua/cemento $r_{\mathbb{N}}$ ' del mortero prescrito por la Norma N'. 


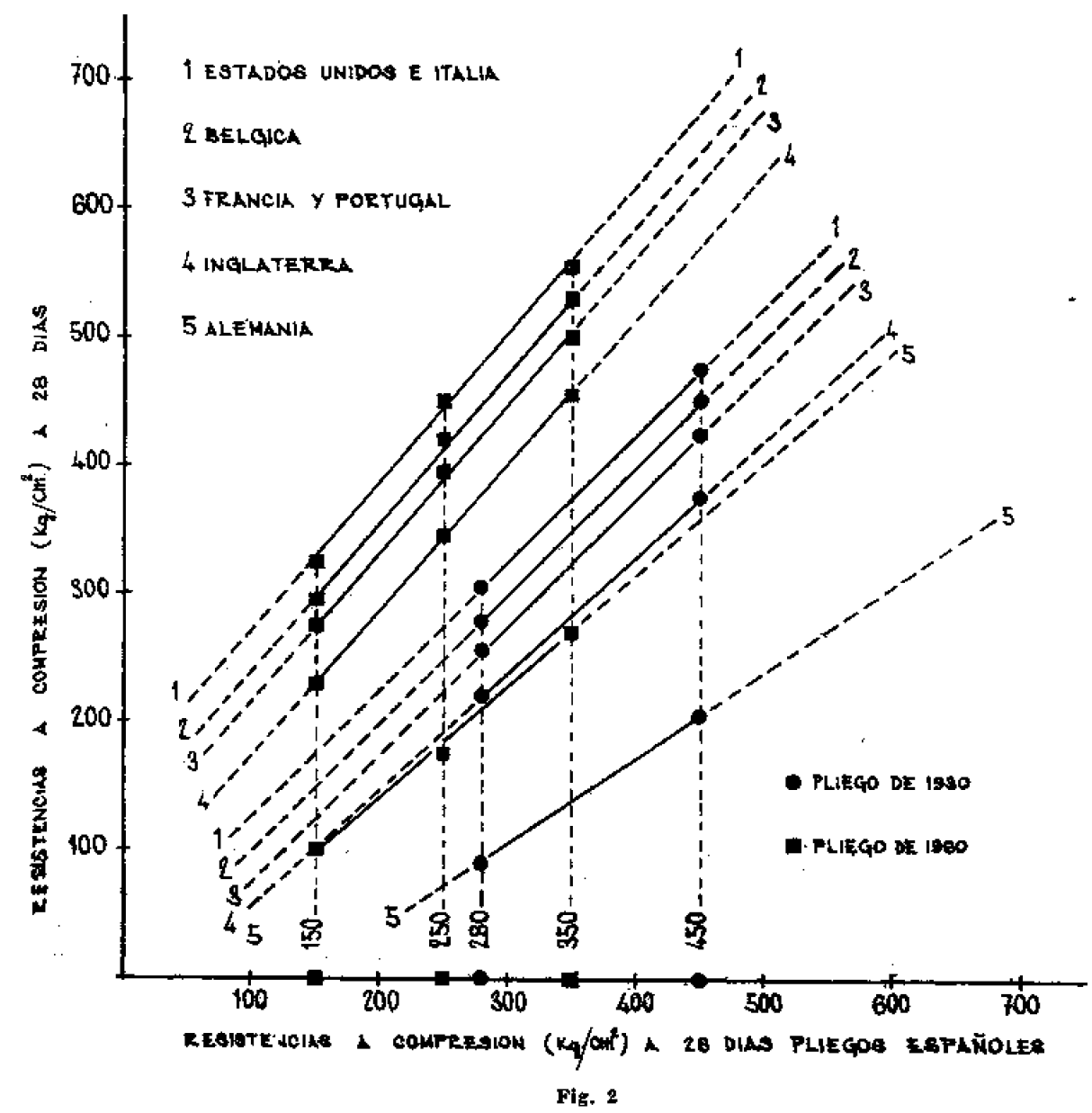

Si la Norma de cuyos datos se supone que se parte es una extranjera $\mathrm{N}^{\prime}$, recíprocamente se tienen las dos ecuaciones:

$$
\begin{aligned}
\log \mathrm{R}_{\mathrm{A}} & =3,10517-\frac{0,34}{r_{\mathrm{N}^{\prime}}}\left(3,10517-\log \mathrm{R}_{\mathrm{N}^{\prime}}\right) \\
\log \mathrm{R}_{\mathrm{M}} & =3,10517-\frac{0,50}{r_{\mathrm{N}^{\prime}}}\left(3,10517-\log \mathrm{R}_{\mathrm{N}^{\prime}}\right)
\end{aligned}
$$

que permiten calcular en cada caso la resistencia de un cemento según uno cualquiera de los Pliegos españoles, $\mathrm{R}_{\mathrm{A}}$ ó $\mathrm{R}_{\mathrm{M}}$, cuando se conoce su resistencia $\mathrm{R}_{\mathrm{N}^{\prime}}$ en una Norma extranjera $\mathrm{N}^{\prime}$ y la relación agua/cemento $r_{\mathrm{N}^{\prime}}$ del mortero de ensayo prescrito por ésta.

Tiene interés calcular, a partir de las ecuaciones [VI] y [VII], y para algunas Normas extranjeras, las resistencias que según ellas corresponden a las mínimas prescritas por los Pliegos españoles. Si se toman en consideración a estos efectos las Normas alemanas, belgas, británicas, estadouniden- 
CUADR

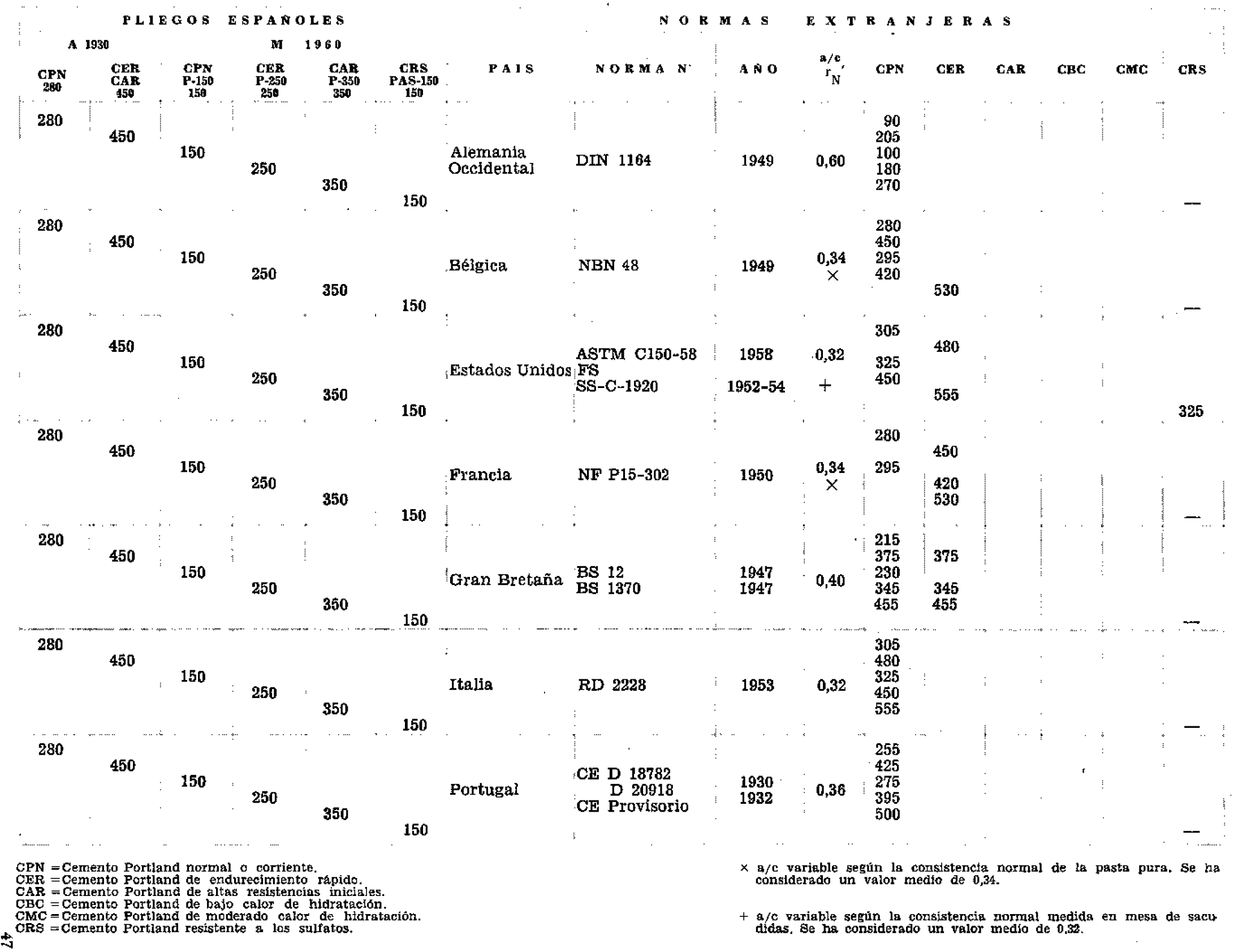


C UA D R O

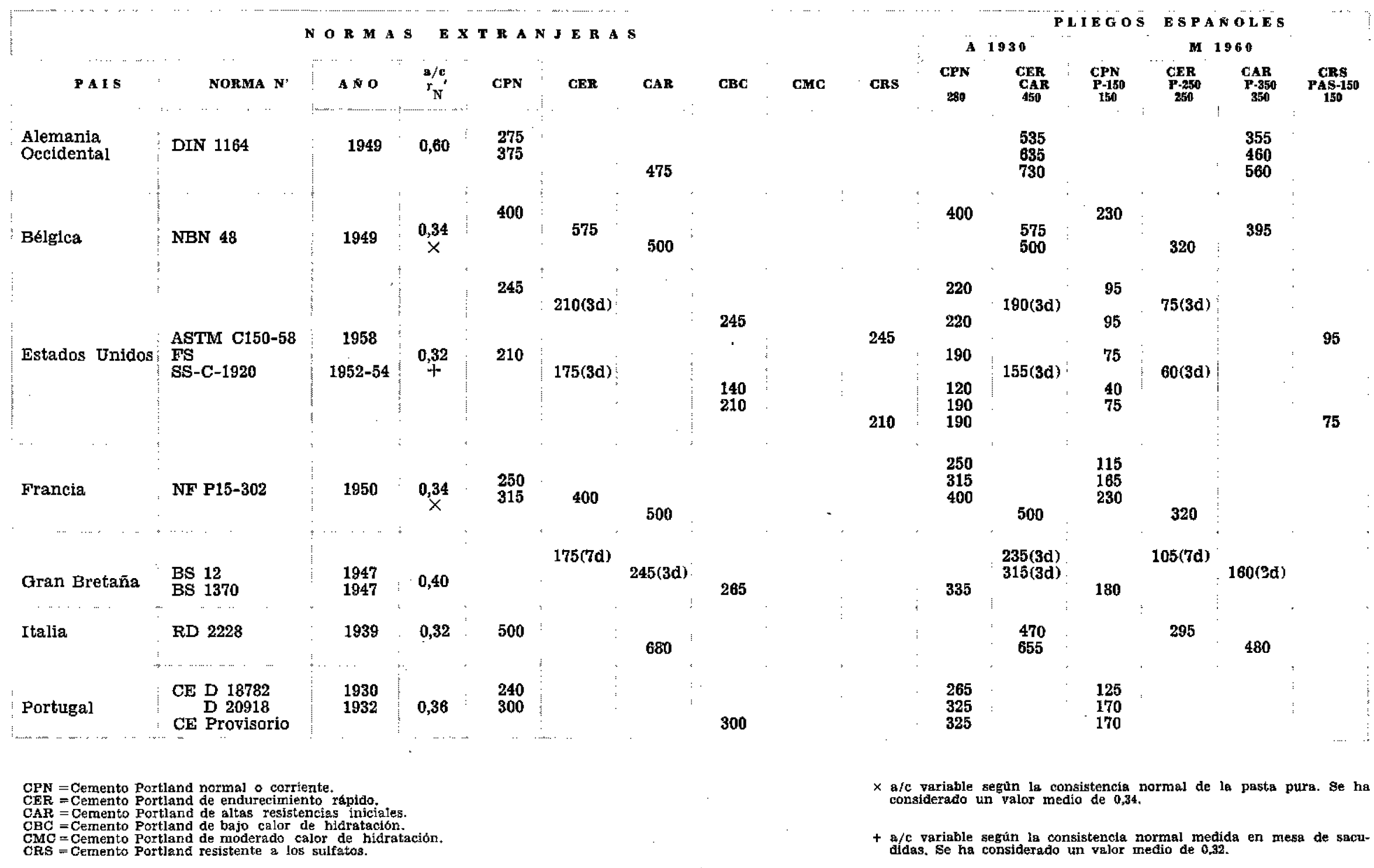



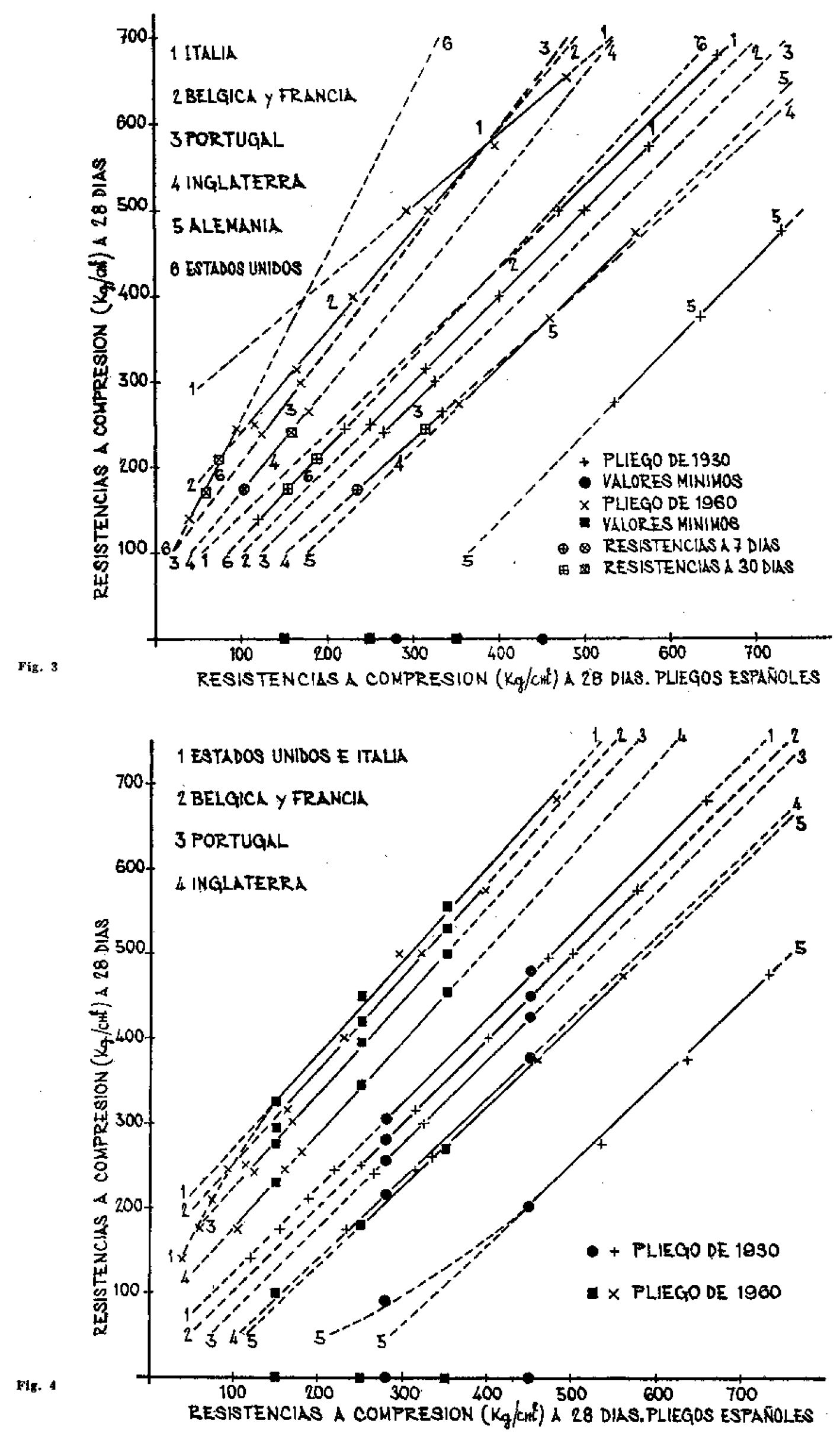
ses, francesas, italianas y portuguesas, las equivalencias resultantes son las indicadas en el cuadro 3 , donde los valores están redondeados a 0 ó 5.

En el gráfico de la figura 2 están representadas las equivalencias del cuadro 3.

Igualmente tiene interés calcular, a partir de las ecuaciones [VIII] y [IX],y para los Pliegos españoles de 1930 y 1960 , las resistencias que según ellos corresponden a las mínimas prescritas por las Normas extranjeras citadas antes. Las equivalencias resultantes son las indicadas en el cuadro 4 , donde los valores están redondeados a 0 ó 5 .

En el gráfico de la figura 3 están representadas las equivalencias del cuadro 4.

El gráfico de la figura 4, que reúne los datos de las 2 y 3 , da una visión más completa de las equivalencias calculadas.

Al aplicar las fórmulas establecidas en $3,3.1$ y 3.2, es preciso tener en cuenta que a veces no se conoce el valor de $r_{\mathrm{N}}{ }^{\prime}, \dot{o}$ bien éste es variable según la consistencia normal de la pasta de cemento o según la plasticidad del mortero de ensayo (seco, plástico o flúido). Se elige entonces un valor medio y la resistencia obtenida por cálculo se aproximará más o menos a la que se obtendría experimentalmente, según el acierto en la elección.

En general, puede decirse que variaciones del orden de $\pm 0,02$ respecto del valor verdadero de la relación agua/cemento, en el intervalo muy frecuente de valores comprendidos entre 0,32 y 0,36 (valor medio 0,34), suponen, «grosso modo», unas diferencias medias entre las resistencias resultantes, del orden de $\pm 5 \%$ a $\pm 10 \%$, en todo el campo de valores que presentan éstas en los casos más frecuentes.

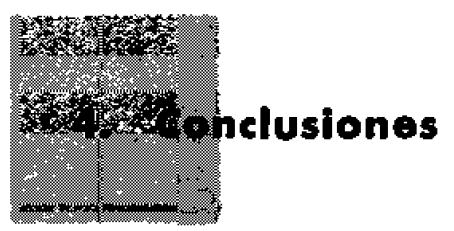

Puede afirmarse, en general, que al calcular por las fórmulas establecidas las equivalencias, tanto entre los Pliegos españoles antiguo y modemo, como entre cada uno de éstos y cualquier Norma extranjera, o viceversa, no son probables diferencias superiores al 10 ó $15 \%$ entre los valores calculados y los que se obtendrían experimentalmente.

Las equivalencias calculadas, por lo que respecta a los dos Pliegos españoles, ponen de relieve que el cemento Portland normal del Pliego de 1930 es algo inferior al P-150 del Pliego de 1960; que el supercemento del Pliego de 1930 es algo superior al P-250 del Pliego de 1960; y que el P-350 de éste corresponde a un buen supercemento del Pliego de 1930, por cuanto que daría, según éste, una resistencia de unos $530 \mathrm{~kg} / \mathrm{cm}^{2}$.

Por otra parte, con relación a las Normas norteamericanas ASTM, el P-150 es análogo al tipo I, el P-350 equivalente al tipo III, y el P-250 ocupa un lugar intermedio. 